\title{
Neurosyphilis in a Non-HIV Patient: More than a Psychiatric Concern
}

\author{
Michael K. Tso, Kevin Koo* and Grace Y. Tso
}

\begin{abstract}
Neurosyphilis is a form of tertiary syphilis infection caused by the spirochete bacterium Treponema pallidum. Patients suffering from this illness can present with neurological manifestations such as headaches, seizures, hearing loss, and ataxia. However, the typical presentation of neurosyphilis is the insidious onset of psychiatric symptoms including personality changes. A good history and clinical work-up is essential in the diagnostic process. There has been a recent increase in the incidence of infectious syphilis in Canada (1). However, in other parts of the world including China, infectious syphilis rates have remained high due to limited access to primary care and affordable treatments $(2$ Here, we present a case of neurosyphilis in a $\mathbf{4 0}$ year old Chinese male residing in China who presents with an 18 month history of personality changes as well as neurological and physical manifestations of the infection.
\end{abstract}

\section{CASE}

A 40 year old Chinese man residing in China was brought into the emergency room by his family presenting with grandiose delusions, irritability, and memory deterioration, which have become progressively worse in the past 6 months. The patient was hostile, uncooperative, and was not able to provide any personal or medical history. Over the past 18 months, the family had noticed dramatic personality changes that included constricted affect, irritability, and apathy. They had also noted that the patient demonstrated ataxia, dysarthria, and progressive behavioural disturbances such as loss of social graces and impairment of activities of daily living. The family recalled that the patient frequently complained of headaches, vertigo, and non-specific abdominal pain in the past 6 months. There was no known history of trauma, malignancy, or vascular disease. He had developed urinary incontinence 3 weeks ago. The patient had no known history of substance abuse or chronic alcohol use. He was not on any medications. There was no personal or family history of psychiatric

*To whom correspondence should be addressed:

Kevin Koo

University of Toronto

Medical Sciences Building, Rm 2171A, 1 King's College Circle

Toronto, Ontario, Canada

M5S1A8

Telephone: 647-262-1335

Email: kevin.koo@utoronto.ca illness. According to the family, the patient was heterosexual and had been single for at least the past 8 months. It was unknown whether he had a history of a sexually-transmitted infection or any previous genital manifestations. The family believes he had been sexually-active since his early twenties, but it is unclear as to the number of sexual partners or the use of condoms. The patient's mood was highly irritable with blunted affect. He denied having any suicidal or homicidal ideation and there was no evidence of perceptual disturbances. He was admitted for further evaluation and treatment by neurology and clinical psychiatry.

\section{PHYSICAL EXAMINATION}

The physical examination was remarkable for neurological findings but was otherwise normal. The patient was afebrile and vital signs were within normal limits. Neurological examination revealed a wide-based gait and ataxia. Pupils in both eyes responded to accommodation and convergence, but they were not reactive to light (Argyll Robertson pupils). Cranial nerve exam was otherwise unremarkable. No lateralizing signs were present. The patient had cognitive impairment and grandiose delusions. $\mathrm{He}$ appeared withdrawn and apathetic. The Mini-mental status examination (MMSE) score was 7/30. MMSE score and psychiatric symptoms were compatible with severe cognitive impairment and dementia. 


\section{INVESTIGATIONS}

Hematological tests were unremarkable. Hepatitis B and $\mathrm{C}$ as well as Human Immunodeficiency Virus (HIV) tests were all negative. Both Treponema Pallidum Agglutination Assay (TPPA) and Rapid Plasma Reagin (RPR) yielded positive serum results (RPR serum titre 1:64). Urine toxicology screen was negative. Chest Xray and electrocardiogram were normal. Brain MRI revealed diffuse frontocortical atrophy. A lumbar puncture revealed a transparent, colourless cerebrospinal fluid (CSF) with protein of $98 \mathrm{mg} / \mathrm{dL}$ (normal: $20-45 \mathrm{mg} / \mathrm{dL}$ ), white blood cell count of $21 / \mu \mathrm{l}$ (normal: $\leq 5 \mu \mathrm{l}$ ), and glucose of $3.75 \mathrm{mmol} / \mathrm{L}$ (normal: 2.5-4.4mmol/1). CSF-RPR and CSF-TPPA were both positive (CSF-RPR titre 1:8).

\section{TREATMENT AND MANAGEMENT}

Based on the investigations, the patient was diagnosed with neurosyphilis and was treated with Penicillin G 4 million units IV every 4 hours for 14 days, followed by Penicillin G procaine 2.4 million units IM daily and probenecid $500 \mathrm{mg}$ by mouth four times dailyfor 14 days. His MMSE score before and 8 weeks after treatment were $7 / 30$ and $21 / 30$ respectively. He regained bladder control 6 days after the start of treatment. The patient was discharged after 8 weeks and is currently being monitored by neurology and psychiatry for at least the next 2 years. Syphilis serology will be performed regularly during that time.

\section{DISCUSSION}

In Canada, the reported rate of infectious syphilis was an all-time low of 0.4/100,000 in 1997 with a dramatic rise to $3.5 / 100,000$ in 2004 (1). The reason for this increase is unclear, although it appears to be associated with unsafe sexual practices especially in men who have sex with men (MSM), illicit intravenous drug use, and concurrent HIV infection (3). Unfortunately, data regarding the incidence of neurosyphilis in Canada is not available. In a systematic review of China's syphilis seroprevalence from 2000 to $2005,660 / 100,000$ of premarital individuals were reported to have both a positive screening nontreponemal test (i.e. RPR, Venereal Disease Research Laboratory (VDRL)) and a positive treponemal-based confirmatory test (i.e. Treponema Pallidum Agglutination Assay) (2). In select high-risk groups in China, incarcerated female sex workers, drug users, and MSM had syphilis seroprevalence rates of $12.49 \%, 6.81 \%$, and $14.56 \%$, respectively (2). New infections can be vertically transmitted from mother to baby or more typically, acquired by sexual contact (4). Syphilis also enhances the transmission of HIV by 3 to 5 times due to inflammation in the genital area and the association with high risk behaviours (5).

Syphilis is caused by the spirochete bacteria Treponema pallidum. The clinical course can be divided into several distinct stages: primary, secondary, early latent, late latent and tertiary stages (6). Primary syphilis manifests as a painless chancre at the site of inoculation, typically involving the penis, vagina, vulva, cervix, anal canal, perianal region, or mouth. Incubation period ranges from 9 to 90 days from initial exposure and the infection resolves spontaneously within 4-5 weeks if untreated (7). Syphilis may recur systemically as secondary syphilis and is characterized by constitutional symptoms (fever, malaise, anorexia), headache, lymphadenopathy, condyloma lata, mucosal ulceration and a generalized, symmetrical macularpapular rash involving the trunk, soles and palms (6). The patient may experience relapsing episodes of secondary syphilis for several years. After spontaneous resolution, the infection enters an early latent stage in which the patient is asymptomatic but has positive serologic testing and remains highly infectious. Beyond one year of latency, the asymptomatic infection is classified as late latent syphilis. Failure to treat may result in progression to the most serious stage called late symptomatic syphilis or tertiary syphilis (6).

If untreated in the latent phase, approximately $40 \%$ of patients would then develop tertiary syphilis (4). Manifestations of tertiary syphilis include cardiovascular syphilis, gummatous syphilis and neurosyphilis. Cardiovascular syphilis may occur 15-30 years after primary infection and mainly involve large vessels, resulting in proximal aortic aneurysm, aortic regurgitation and angina (7). Gummatous syphilis presents 3-12 years after primary infection as inflammatory fibrous nodules that are locally destructive (7). These lesions can involve any organ of the body, but usually affect skin or bone. Neurosyphilis may be divided into early and late forms, of which only the latter is considered to be tertiary.

Early neurosyphilis commonly affects the CSF, meninges, and vasculature (8). Manifestations include asymptomatic or symptomatic meningitis and meningovascular disease. Less than 2 years after primary infection, patients may present with asymptomatic meningitis with CSF abnormalities including a reactive VDRL, a lymphocytic pleocytosis, and elevated protein. Lack of treatment at this stage may result in progression toward more serious forms of neurosyphilis. Symptomatic meningitis most often presents in the first year after inoculation. Patients typically complain of headache, confusion, nausea/vomiting, stiff neck and decreased visual acuity. Syphilis may also cause inflammation of the intracranial vasculature and must be part of the 
differential diagnosis for an ischemic stroke in a young patient. Meningovascular disease may be visualized on angiography showing focal narrowing or occlusion (9).

Common forms of late neurosyphilis affect CNS parenchyma and include general paresis of the insane (also known as general paresis) and tabes dorsalis (8). Both forms have poor prognoses without treatment but are very uncommon in the antibiotic era. General paresis develops 10-25 years after initial infection and manifests as a progressive dementia with symptoms of personality change and poor memory (7). Common abnormal neurologic findings include facial and limb hypotonia, dysarthria, and intention tremor. Patients may exhibit psychiatric symptoms such as depression, mania, or psychosis and may inadvertently be admitted to psychiatry. Tabes dorsalis presents with ataxia and lancinating pains secondary to destruction of the posterior columns and dorsal roots of the spinal cord. The pain affects the limbs, trunk and face and described as sudden, brief and severe. Characteristic ArgyllRobertson pupils may be present - the pupils respond to accommodation and convergence but do not react to light. Other signs and symptoms include paresthesias, severe episodic nausea/vomiting, impaired vibration sense and proprioception, and absent lower extremity reflexes (8).

The diagnosis of neurosyphilis in particular relies heavily on clinical exam, CSF analysis, and risk factors including positive HIV status, multiple sexual partners, illicit IV drug use, MSM, and a previously documented syphilis infection. If a genital lesion is present, darkfield microscopy may reveal spirochetes moving with a spiralling motion (10). Systemic syphilis is detected by screening serum nontreponemal tests (VDRL, RPR) and confirmatory treponemal tests (fluorescent treponemal antibody absorption, FTA-ABS and Treponemal pallidum particle agglutination assay, TPPA). Late neurosyphilis may have nonreactive serum nontreponemal tests. CSF-VDRL is the gold standard for diagnosing neurosyphilis, although it is not as sensitive as the less-specific CSF FTA-ABS (11). CSF demonstrating lymphocytic pleocytosis, high WBC count, and a high protein concentration is consistent with neurosyphilis. In a recent, multi-center prospective study, it was found that patients with serum RPR titres $>=1: 32$ were highly predictive of also having neurosyphilis (defined as a positive CSF-VDRL or CSF $\mathrm{WBC}>20$ cells/microliter), regardless of syphilitic stage, HIV status, or previous non-neurosyphilis treatment (12). In HIV-positive patients infected with syphilis, serum RPR titres may be elevated in early HIV infection compared to HIV-negative patients. In late stage HIV infection, serologic response to syphilis may be delayed or absent. These paradoxical findings may be attributed to B-cell hyperfunctioning and failure, respectively (13).

The patient in question presented with an insidious onset of personality change, delusions, and memory loss. The differential diagnosis of these psychiatric symptoms in a 40 year old male includes psychiatric conditions such as schizophrenia, bipolar disorder, and early-onset Alzheimer's dementia as well as infectious causes such as HIV dementia, CNS tuberculosis, herpes encephalitis, and neurosyphilis, among others. As neurosyphilis is often called "the great masquerader," it is critical to have this diagnosis on the differential of any patient presenting with insidious onset of psychiatric symptoms with atypical features. Although possible, forty years of age is fairly old for new onset schizophrenia and too young for Alzheimer's. However, despite the absence of syphilis risk factors outlined above, the presence of frequent headaches and the characteristic Argyll-Robertson pupils in this patient strongly supported the diagnosis of neurosyphilis even before formal investigations were carried out.

The standard of treatment for neurosyphilis is Penicillin G 3-4 million units IV q4h or 24 million units continuous IV infusion for 10-14 days (14). Penicillin G procaine 2.4 million units IM qd plus probenecid $500 \mathrm{mg}$ po qid for 10-14 days may be given as an alternative (15). Ceftriaxone $2 \mathrm{~g}$ IV qd for $10-14$ is also an option (14). Follow-up clinical examination and lumbar puncture should be scheduled at 3-6 months after treatment and every 6 months thereafter until the CSF-VDRL becomes nonreactive and the CSF white blood cell count normalizes (8). CSF abnormalities clear typically in 2 years. Prognosis is excellent with early treatment initiation, but can be poor with irreversible brain damage.

\section{SUMMARY}

Untreated syphilis can progress to a form of tertiary syphilis called neurosyphilis. This is a serious concern as the patient begins to demonstrate marked cognitive and/or behavioural impairment as well as many other neurological and physical symptoms. Clinical suspicion of neurosyphilis can be made based on a detailed medical history and physical exam including a history of high-risk behaviours, previous documented syphilis infection, insidious onset of psychiatric symptoms, headaches and characteristic neurological findings such as Argyll-Robertson pupils. The diagnosis can be confirmed with serum non-treponemal tests such as VDRL and RPR, serum treponemal tests such as FTAABS and TPPA, and neurosyphilis-specific tests, such as CSF-RPR and CSF-VDRL. The outcome of patient with neurosyphilis is highly dependent on the amount of brain damage and the extent of disability before the 
initiation of treatment.

\section{ACKNOWLEDGMENT}

The authors would like to sincerely thank Dr. Hui Li for his support and encouragement in the preparation of this case report.

\section{REFERENCES}

1. Public Health Agency of Canada. 2004 Canadian Sexually Transmitted Infections Surveillance Report. CCDR 2007; 33S1:1-69.

2. Lin CC, Gao X, Chen, X-S, Chen Q and MS Cohen. China's Syphilis Epidemic: A Systematic Review of Seroprevalence Studies. Sexually Transmitted Diseases. December 2006; 33(12):726-736.

3. Goldon MR, Marra CM and KK Holmes. Update on Syphilis: Resurgence of an old problem. JAMA 2003; 290:1510-1514.

4. Goh BT. Syphilis in Adults. Sexually Transmitted Infections 2005; 81: 448-452.

5. Wasserheit JN. Epidemiological synergy: Interrelationships between human immunodeficiency virus infection and other sexually transmitted diseases. Sexually Transmitted Diseases. March-April 1992; 19(2):61-77.

6. LaFond RE and SA Lukehart. Biological Basis for Syphilis. Clin Microbiol Rev. 2006 January; 19(1): 29-49.

7. French P. Clinical Review: Syphilis. BMJ 2007; 334:143-7.

8. Mandell GL, Bennett JE and R Dolin. Mandel, Douglas, and Bennett's: Principles and Practice of Infectious Diseases. 6th edition. Churchill Livingstone. 2005. Chapter 235.

9. Cohen J, Powderly WG, et al. Cohen \& Powderly: Infectious Diseases. 2nd edition. Mosby. 2004. Chapter 75.

10. Birnbaum NR, Goldschmidt RH, and WO Buffett. Resolving the common clinical dilemmas of syphilis. Am Fam Physician. 1999 April; 59(8):2233-40, 2245-6.

11. Jaffe HW, Larsen SA, Peters M, et al. Tests for treponemal antibody in CSF. Arch Intern Med 1978; 138:252.

12. Marra CM, Maxwell CL, Smith SL, et al. Cerebrospinal fluid abnormalities in patients with syphilis: association with clinical and laboratory features. J Infect Dis 2004; 189: 369-79.

13. Hicks CB. Serologic testing for syphilis. Uptodate.com. 2006.

14. Drugs for Sexually transmitted diseases. Treat Guidel Med Lett $2004 ; 2: 67$

Kevin Koo $(\mathrm{BScH})$ is the outgoing Vice-President External Affairs of University of Toronto Medical Society and the President of Class of 2010. He received a B.Sc (Hons) in chemistry and mathematics from Queen's University. He is currently a third year medical student at the University of Toronto.

Michael Tso received a B.Sc (Hons) in Life Sciences from Queen's University. He is currently a fourth year medical student at the University of British Columbia.

Grace Tso $(\mathrm{BScH})$ is the outgoing President of the Queen's University Pre-Medical Society. She received a B.Sc (Hons) in Life Sciences from Queen's University. She is now entering her first year as a medical student at the University of Queensland in Australia. 